\title{
Damage of Metallic Materials during High-Temperature Creep
}

\author{
A.R. Arutyunyan, R.A. Arutyunyan, R.R. Saitova \\ St. Petersburg State University, 198504, Russia, \\ a.arutyunyan@spbu.ru, rigastr@yandex.ru
}

\begin{abstract}
In the conditions of high-temperature creep, the evolution of damage of metallic materials is occurred. For its description, the damage conception of Kachanov-Rabotnov is used. In this paper the damage parameter is defined as the relative changes of the material density, which is an integral measure of the damage. Taking into account this parameter and the mass conservation law, interrelated kinetic equations for creep deformation and damage parameter are formulated.
\end{abstract}

Keywords: high-temperature creep, long-term strength, damage conception, density changes.

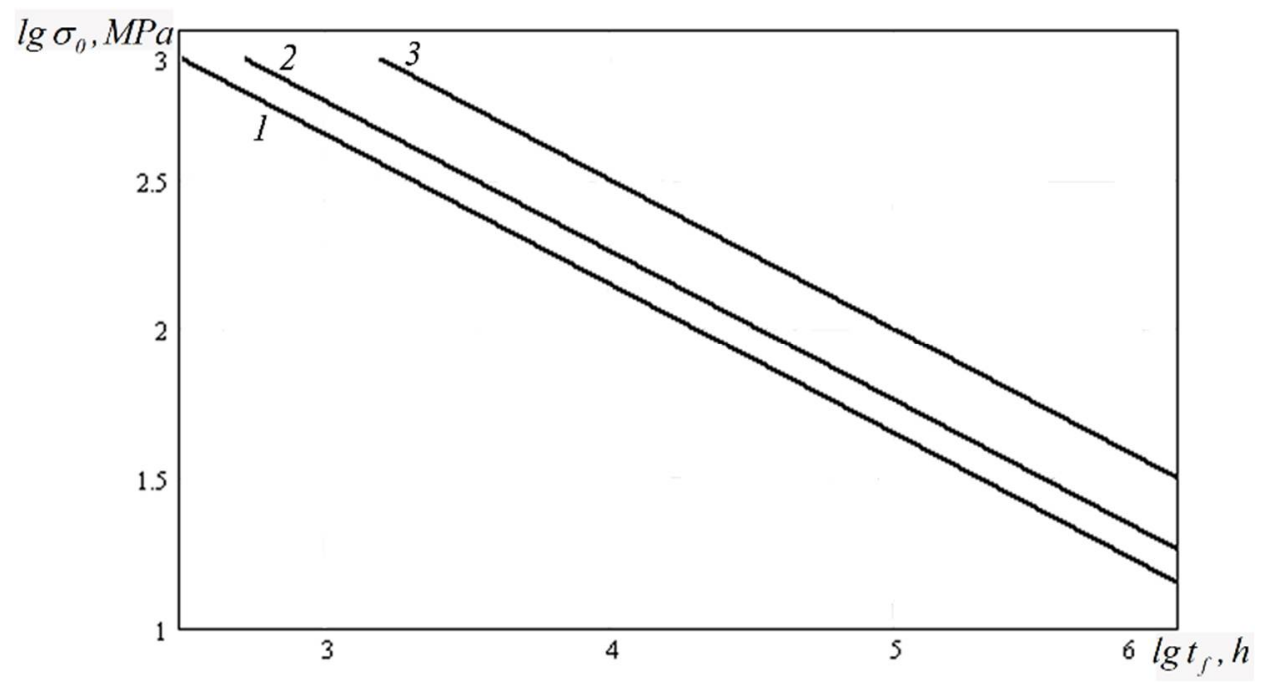

Theoretical long-term strength curves for different values of parameter $\alpha$ : $\alpha=6$ (curve 1), $\alpha=4$ (curve 2) and $\alpha=2$ (curve 3) 


\title{
Поврежденность металлических материалов в процессе высокотемпературной ползучести
}

\author{
А.P. Арутюнян, Р.А. Арутюнян, Р.P. Саитова \\ Санкт-Петербургский государственный университет, \\ Россия, Санкт-Петербург, 198504, Петергоф, Университетский пр., 28 \\ a.arutyunyan@spbu.ru; rigastr@yandex.ru
}

\begin{abstract}
Аннотация
В условиях высокотемпературной ползучести происходит эволюция поврежденности металлических материалов. Для ее описания используется концепция поврежденности Качанова-Работнова. В работе параметр поврежденности определяется как относительное изменение плотности материала, которая является интегральной характеристикой поврежденности.
\end{abstract}

Ключевые слова: высокотемпературная ползучесть, длительная прочность, концепция поврежденности, относительное изменение плотности.

\section{1. Введение}

В общей постановке концепция поврежденности (сплошности) и хрупкого разрушения рассматривалась в работах $[1,2]$. Принимается во внимание, что реальные материалы имеют случайную структуру, поэтому параметр поврежденности считается статистическим показателем. Согласно представлениям статистической физики, скорость изменения параметра сплошности зависит от напряжения и параметра сплошности.

Основные положения концепции хрупкого разрушения Качанова-Работнова основываются на данной гипотезе и конкретизируются в виде степенной зависимости от напряжения и параметра сплошности. В модели Л.М. Качанова [3] вводится параметр сплошности $\psi$, $1 \leq \psi \leq 0$, в модели Ю.Н. Работнова [4,5] рассматривается параметр поврежденности $\omega=1-\psi, 0 \leq \omega \leq 1$, который задается соотношением $\omega=F_{T} / F_{0}$, где $F_{T}$ - площадь трещин, располагающихся к моменту времени $t$ в поперечном сечении растягиваемого образца, $F_{0}-$ начальная площадь поперечного сечения образца.

Для учёта деформационных процессов Работнов ввел систему из двух взаимосвязанных уравнений для деформации ползучести $\varepsilon$ и параметра поврежденности $\omega$ [5]

$$
\begin{aligned}
& \frac{\mathrm{d} \varepsilon}{\mathrm{d} t}=b \sigma^{m}(1-\omega)^{-q}, \\
& \frac{\mathrm{d} \omega}{\mathrm{d} t}=c \sigma^{n}(1-\omega)^{-r},
\end{aligned}
$$

где $b, c, m, n, q, r$ - постоянные.

В случае чисто хрупкого разрушения и малых деформаций считается, что $F=F_{0}$, $\sigma=\sigma_{0}$ и из решения системы (1)-(2) при начальных условиях $t=0, \varepsilon=0, \omega=0$ Работнов получил соотношение для деформации ползучести, которое считается основным результатом в теории Работнова. Данное соотношение описывает третий участок кривой ползучести, который в области хрупких разрушений полностью определяется поврежденностью материала. 


\section{2. Кинетические уравнения для параметра поврежденности и деформации ползучести.}

В работах $[6,7]$ предложена система уравнений для скорости ползучести и поврежденности, основанная на параметре сплошности $\psi=\rho / \rho_{0}$, где $\rho_{0}-$ начальная, $\rho$ - текущая плотность образца. В начальном состоянии $\rho=\rho_{0}, \omega=0, \psi=1$, в момент разрушения $\rho=0$, $\omega=1, \psi=0$. В данной работе представлен модифицированный вариант этих уравнений

$$
\begin{gathered}
\frac{\mathrm{d} \varepsilon}{\mathrm{d} t}=B \sigma_{0}^{m}\left(\frac{\rho}{\rho_{0}}\right)^{m-\beta} e^{m \varepsilon}, \\
\frac{\mathrm{d} \rho}{\mathrm{d} t}=-\rho_{0} A \sigma_{0}^{n}\left(\frac{\rho}{\rho_{0}}\right)^{n-\alpha} e^{n \varepsilon}
\end{gathered}
$$

где $A, B, \alpha, \beta$-постоянные.

Аналитическое решение системы уравнений (3)-(4) в общем виде не представляется возможным. Рассмотрим некоторые приближённые решения. Если ограничиться случаем малых деформаций, можно считать $e^{n \varepsilon} \approx 1, e^{m \varepsilon} \approx 1$, и из решения уравнений (3)-(4) при начальных условиях, соответственно, $t=0, \varepsilon=0$ и $t=0, \psi=1$ следуют соотношения для деформации ползучести и изменения плотности

$$
\begin{gathered}
\varepsilon(t)=\frac{B \sigma_{0}^{m-n}}{A \gamma}\left\{1-\left[1-(\alpha-n+1) A \sigma_{0}^{n} t\right]^{\frac{\gamma}{\alpha-n+1}}\right\}, \\
\rho(t)=\rho_{0}\left[1-(\alpha-n+1) A \sigma_{0}^{n} t\right]^{\frac{1}{\alpha-n+1}},
\end{gathered}
$$

где $\gamma=m-\beta+\alpha-n+1$.

С учётом условия разрушения $t=t_{f}, \rho=\rho_{*}$ из (6) следует критерий длительной прочности в виде

$$
t_{f}=\frac{1-\left(\rho_{*} / \rho_{0}\right)^{\alpha-n+1}}{(\alpha-n+1) \cdot A \sigma_{0}^{n}},
$$

На рис. 1 показаны теоретические кривые длительной прочности согласно уравнению (7) при различных значениях параметра $\alpha: \alpha=6$ - кривая $1, \alpha=4$ - кривая 2 и $\alpha=2-$ кривая 3. При расчетах приняты следующие значения коэффициентов: $\rho_{*}=0.9 \rho_{0}$, $A=1 \times 10^{-9}[\mathrm{M \Pi a}]^{-2}, n=2$.

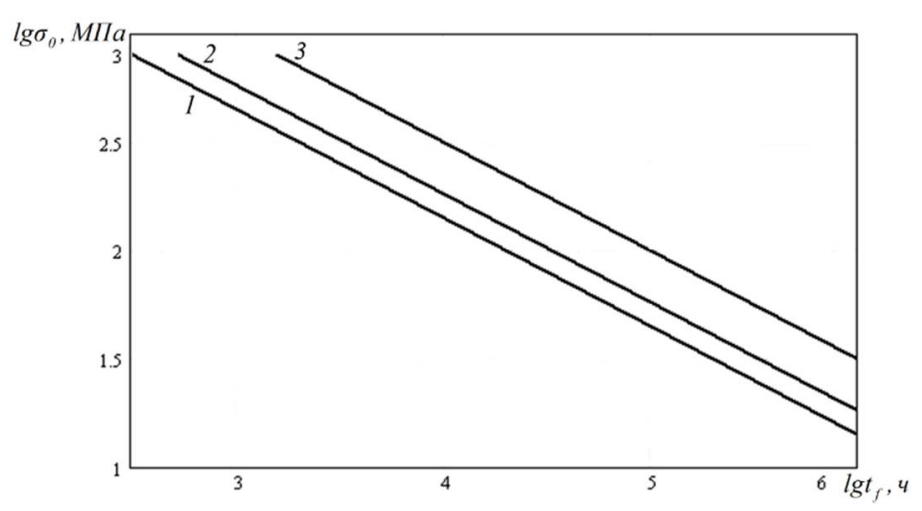

Рис. 1. Теоретические кривые длительной прочности согласно критерию (7) при различных значениях $\alpha: \alpha=6$ - кривая 1 , $\alpha=4-$ кривая 2 и $\alpha=2-$ кривая 3 
Критерий Качанова- Работнова (кривая 2) является частным случаем полученного критерия. Из рис. 1 видно, что теория Качанова-Работнова предсказывает завышенные значения времени до разрушения и накопление деформации ползучести в этом случае происходит более интенсивно по сравнению с критерием (7).

\section{3. Заключение}

В работе в качестве параметра сплошности рассматривается относительное изменение плотности, которая является интегральной мерой накопления структурных микродефектов. С учетом этого параметра и закона сохранения массы сформулированы взаимосвязанные кинетические уравнения для деформации ползучести и параметра поврежденности. Получены аналитические решения этих уравнений и сформулирован критерий длительной прочности. Построены соответствующие теоретические кривые.

\section{Благодарности и ссылки на гранты}

Работа выполнена при финансовой поддержке Российского фонда фундаментальных исследований (грант № 18-01-00146).

\section{Литература}

1. Haward R.N. The Extension and Rupture of Cellulose Acetate and Celluloid// Trans. Farad. Soc. 1942. V. 38. Pp. 394-400.

2. Бокшицкий М.Н. Длительная прочность полимеров. М.: Химия, 1978. 310 с.

3. Качанов Л.М. О времени разрушения в условиях ползучести // Изв. АН СССР. ОТН. 1958. № 8. C. $26-31$.

4. Работнов Ю.Н. О механизме длительного разрушения. Вопросы прочности материалов и конструкций. М.: Изд-во АН СССР, 1959. С. 5-7.

5. Работнов Ю.Н. Ползучесть элементов конструкций. М.: Наука, 1966. 752 с.

6. Arutyunyan R.A. High-Temperature Embrittlement and Long-Term Strength of Metallic Materials // Mech. Solids. 2015. V. 50. I. 2. Pp. 191-197.

7. Arutyunyan A., Arutyunyan R., Saitova R. The Criterion of High-Temperature Creep of Metals Based on Relative Changes of Density. // WSEAS Transactions on Applied and Theoretical Mechanics. 2019. 14. Pp. 140-144.

Статья поступила в редакцию 15 сентября 2019 г. 